\title{
A clinical comparative study on the effects of metformin and pioglitazone on clinical symptoms in cases of polycystic ovarian syndrome
}

\author{
Sumita Tanwar $^{1 *}$, Guru Das Khilnani ${ }^{2}$
}

\begin{abstract}
${ }^{1}$ Department of Pharmacology S.P. Medical College Bikaner, Rajasthan 334001,

${ }^{2}$ Department of Pharmacology, Gujarat Medical Education and research society (GMERS) Medical College; Dharpur, Patan Gujarat
\end{abstract}

Received: 14 October 2015

Revised: 25 November 2015

Accepted: 18 December 2015

*Correspondence to:

Dr. Sumita Tanwar,

Email: sumitavtanwar

@ rediffmail.com

Copyright: (C) the author(s), publisher and licensee Medip Academy. This is an open-access article distributed under the terms of the Creative Commons Attribution Non-Commercial License, which permits unrestricted non-commercial use, distribution, and reproduction in any medium, provided the original work is properly cited.

\begin{abstract}
Background: The present study was aimed to evaluate the efficacy of pioglitazone and comparing the efficacy of pioglitazone with metformin in improvement of clinical characteristics of the PCOS patients.

Methods: The present study was a prospective, parallel group trial in 40 proven cases of PCOS, all the patients underwent clinical evaluations at baseline that include height, weight, BMI, waist to hip ratio (WHR) and hirsutism (FG score). There were two groups in this study group A (unmarried) and group $B$ (married) each divided in two subgroups each having 10 patients, $\mathrm{A}_{1}$ (unmarried - metformin), $\mathrm{A}_{2}$ (unmarried pioglitazone), $\mathrm{B}_{1}$ (married - metformin), $\mathrm{B}_{2}$ (married - pioglitazone) therapy was given for six months with regular clinical evaluation of the patients after every two months. WHR (Waist to hip ratio) was measured according to World Organization Criteria (2003), BMI was calculated by using Quetelets Index formula WHO (1995), the grade of hirsutism was detected using Ferriman-Gallwey score (1961).

Results: On comparing metformin and pioglitazone it was observed that both the drugs equally decreased the FG score; metformin significantly decreased the BMI and WHR whereas pioglitazone significantly increased the BMI and WHR.

Conclusions: Pioglitazone significantly increased the BMI and WHR, whereas it significantly decreased FG score. On comparing metformin and pioglitazone it was observed that both the drugs equally decreased the FG score, metformin significantly decreased the BMI and WHR whereas pioglitazone significantly increased the BMI and WHR.
\end{abstract}

Keywords: BMI, WHR, FG score

\section{INTRODUCTION}

Polycystic ovarian syndrome is one of the common abnormality in reproductive aged women, occurring globally in $6 \%$ to $10 \%$ of the population. ${ }^{1,2}$ This syndrome has over years, been defined in different ways. Polycystic ovarian syndrome (PCOS): a syndrome complex of amenorrhea and hirsutism in women with bilaterally enlarged polycystic ovaries. ${ }^{3}$

According to revised guidelines of PCOS consensus workshop group 4: A women to be diagnosed with PCOS must have two of the following three manifestations; irregular/absent ovulation, elevated levels of androgenic hormones and/or enlarged ovaries containing at least 12 follicles each. Polycystic ovaries are defined as those found on ultrasound to contain at least 12 or more follicles measuring 2 to $9 \mathrm{~mm}$ in diameter and/or have an increased volume of $10 \mathrm{ml}$ or greater. Only one ovary fulfilling these criteria is enough to meet the definition of polycystic ovaries. It should be stressed that polycystic ovaries are not a necessary feature of PCOS and that many women with polycystic ovaries do not have PCOS.

The etiopathogenesis of PCOS is not precise and because of diverse nature of findings in PCOS no single factor will explain the entire spectrum of the syndrome. An understanding of the pathogenesis of PCOS substantiates the concept that it seems to be a multigenetic disorder. Clinical studies support an increased frequency of PCOS in first degree relatives of affected women as suggested by a higher prevalence of glucose intolerance, insulin resistance, and hyperandrogenemia. ${ }^{5-6}$ Many candidate genes for PCOS have been proposed. A number of these genes may alter the hypothalamic-pituitary ovarian axis and the others may play a major role in the pathogenesis 
of insulin resistance and the associated phenotype of this heterogeneous disorder. ${ }^{7}$ Recent studies have demonstrated a candidate gene near the insulin receptor gene locus. ${ }^{8}$ In addition, several alternative hypothesis have been proposed for the pathogenesis of PCOS including: ${ }^{9}$

- Hypothalamic - pituitary abnormalities that result in gonadotropin releasing hormone $(\mathrm{GnRH})$ and luteinizing hormone $(\mathrm{LH})$ dysfunction.

- A primary enzymatic defect in ovarian or combined ovarian and adrenal steroidogenesis.

- A metabolic disorder characterized by insulin resistance in conjunction with compensatory hyperinsulinemia that exerts adverse effects on the hypothalamus, pituitary, ovaries and adrenal glands.

There are no pathognomonic characteristics of the syndrome, but the combination of oligomenorrhea and clinical or biochemical finding of androgen excess are most commonly noted. A persistent weight gain of 10 to 15 pounds per year may be noted. As women gain weight, the clinical features of hirsutism, acne, menstrual cyclic and obstructive sleep apnoea frequently worsen. Associated with the weight gain is increased appetite with a tendency to compulsive ingestion of carbohydrate - rich foods. The occurrence of obesity in conjunction with skin and hair manifestation of the syndrome can have a further negative effect on self-esteem and self-image. ${ }^{10}$

The association between hyperinsulinaemia and PCOS was first noted by Burghen et al in 1980 who found a significant positive correlation between insulin, androstenedione and testosterone levels among PCOS women. ${ }^{11}$ It is estimated that $20-40 \%$ of PCOS women have impaired glucose tolerance, a number approximately seven fold higher than the rates in age and weight matched women. ${ }^{12}$ Secondary hyperinsulinaemia is the key factor responsible for the hyperandrogenism characteristics of the syndrome, ${ }^{13}$ which is attributed to an increased stimulation of the activity of the cytochrome P450c $17 \alpha$ in the ovary. ${ }^{14}$

Because insulin resistance is a cardinal feature of PCOS, one therapeutic approach is to use drugs to improve insulin sensitivity and ovarian function. Insulin sensitizing agents improve insulin action by increasing insulin sensitivity thereby decreasing hyperinsulinemia. Since almost all obese PCOS women and more than half of those with normal weight are insulin resistant and present with some degree of fasting or stimulated hyperinsulinemia, the use of insulin sensitizers could therefore be suggested in most patients with PCOS.

Insulin sensitizers are unique in PCOS because they offer both metabolic and gynaecologic benefit. Several studies are done with metformin but pioglitazone which is a member of newer group thiozolidionediones is not well studied. Therefore the present work was planned in the form of a clinical comparative study on the effects of metformin and pioglitazone in cases of PCOS.

\section{METHODS}

The present study is a prospective, parallel group comparative trial in 40 proven cases of PCOS. The study was carried out in department of gynaecology and obstetrics of tertiary level of hospital. There were two groups in this study (group $\mathrm{A}$ and group $\mathrm{B}$ ) each divided in two subgroups (subgroup $A_{1} \& A_{2}$ and subgroup $B_{1}$ \& $\left.\mathrm{B}_{2}\right)$.

Group A included 20 unmarried women with PCOS, who attended the OPD with complaints like menstrual irregularities, weight gain and cosmetic problems. Group A was randomly assigned to metformin $500 \mathrm{mg}$ thrice a day (subgroup $\mathrm{A}_{1}$ ) or pioglitazone $45 \mathrm{mg}$ per day orally (subgroup $\mathrm{A}_{2}$ ).

Group B included 20 married women with PCOS, who attended the OPD with complaints like menstrual irregularities, weight gain and cosmetic problems. Group $\mathrm{B}$ was randomly assigned to metformin $500 \mathrm{mg}$ thrice a day (subgroup $\mathrm{B}_{1}$ ) or pioglitazone $45 \mathrm{mg}$ per day orally (subgroup $\mathrm{B}_{2}$ ).

All the subgroup received continued treatment for 24 weeks.

\section{Evaluation and follow up}

All the patients underwent evaluations at baseline that included height, weight, BMI, waist to hip ratio (WHR) and hirsutism (FG score). Patients were evaluated after every two months, visit $2,3 \& 4$ were performed after 2 , $4 \& 6$ months of the treatment respectively.

\section{Waist to hip ratio (WHR)}

Waist circumference was determined as the minimum circumference between the iliac crest and the lateral costal margin, whereas the hip circumference was determined as maximum circumference over the gluteal region (buttocks), with a soft tape measure which is according to World Health Organization criteria (2003). ${ }^{15}$

Body weight was taken on weighing machine. Height was calculated by height measuring tape. BMI was calculated by using Quetelets Index formula. But for convenience it was calculated with the help of a tables. ${ }^{16}$

Quetelets Index formula:

$$
\mathrm{BMI}=\frac{\text { Weight }(\mathrm{Kg})}{\text { Height }\left(\mathrm{m}^{2}\right)}
$$

Hirsutism - The grade of hirsutism was detected using Ferriman - Gallwey score ${ }^{17}$ by the same observer. The method to assess hirsutism required the visual scoring of 
the extent of terminal hairs in nine body areas, namedupper lip, chin, chest, upper abdomen, lower abdomen, upper back, lower back, thighs \& upper arms. The lower arms and lower legs were not included in the assessment. Each area was scored from 0 to 4 , resulting in a maximum score of 36 . Score < 8 : no hirsutism, score 8 16: low hirsutism, score 17-24: moderate hirsutism, score $>$ 24: severe hirsutism.

All data were presented as mean \pm SD. Within group differences were analysed by the paired student's t test using the formula,

$$
t=\frac{\mathrm{x} \sqrt{\mathrm{n}}}{\mathrm{s}}
$$

Where $\mathrm{x}=$ the mean of the differences of each set of paired observation before \& after

$\mathrm{SD}=$ the standard deviation of the differences

The value of SD was calculated as follows:

$$
S D=\frac{\sqrt{\Sigma\left(\mathrm{d}-\mathrm{d}^{\prime}\right)^{2}}}{\mathrm{n}-1}
$$

$\mathrm{p}<0.05$ was considered significant.

\section{RESULTS}

As shown in Table 1, in unmarried group $A_{1}$ on metformin $500 \mathrm{mg}$ thrice a day orally there was no significant change in the weight of patients, a very significant decrease was observed in the BMI and WHR of the patients at the visit 4, FG score showed very significant decrease at visit 2,3,4.

In unmarried group $\mathrm{A}_{2}$ on pioglitazone $45 \mathrm{mg}$ per day orally there was no significant change in the weight of patients, a significant decrease was observed in the BMI of the patients at the visit 3 and a very significant decrease was observed in the BMI of the patients at the visit 4 . A very significant decrease in WHR was observed at visit 2,3,4. F-G score showed very significant decrease at Visit 2,3,4.

In married group $\mathrm{B}_{1}$ on Metformin $500 \mathrm{mg}$ thrice a day orally there was no significant change in the weight of patients, a very significant decrease was observed in the BMI of the patients at the visit 2,3,4. WHR showed very significant decrease at visit 4 . F-G score showed very significant decrease at visit 2,3,4.

In married group $\mathrm{B}_{2}$ on pioglitazone $45 \mathrm{mg}$ per day orally there was no significant change in the weight of patients, a significant increase was observed in the BMI of the patients at the visit 3 and a very significant increase was observed in the BMI of the patients at the visit 4. A very significant increase in WHR was observed at visit 2,3,4. FG score showed very significant decrease at visit 2,3,4.

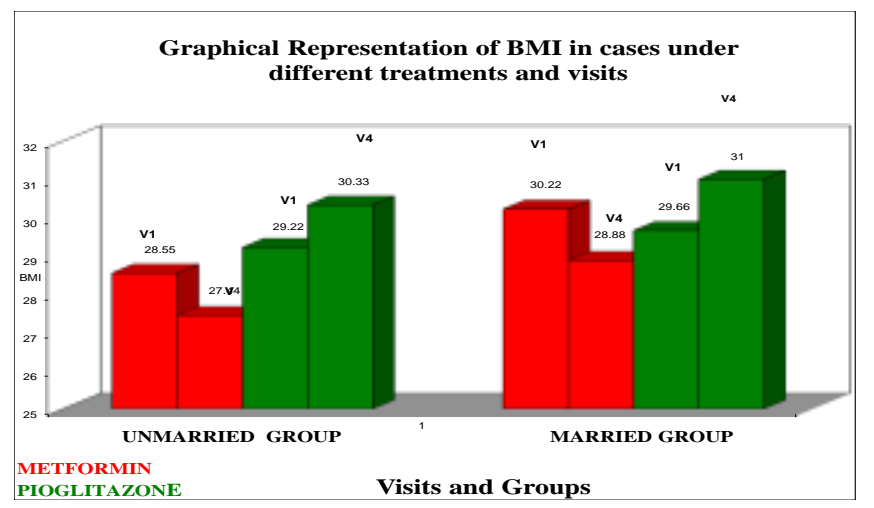

Figure 1: Shows BMI in cases under different treatments and visits.

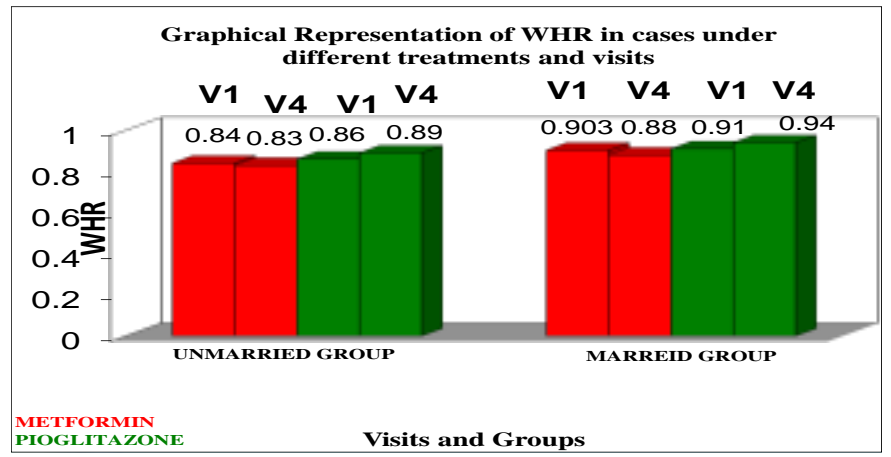

Figure 2: Shows WHR in cases under different treatments and visits.

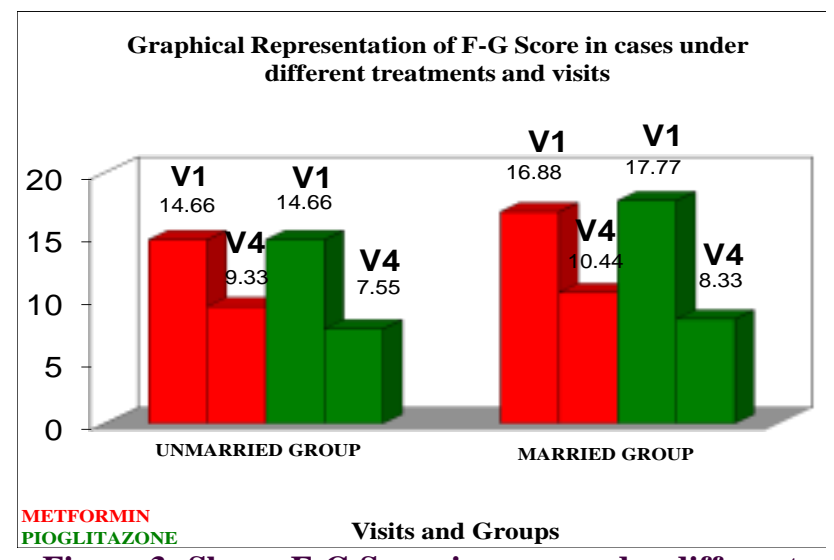

Figure 3: Shows F-G Score in cases under different treatments and visits. 
Table 1: Effects of metformin and pioglitazone on main clinical characteristics of PCOS.

\begin{tabular}{|c|c|c|c|c|c|c|c|}
\hline Parameters & Visit 1 & Visit 2 & $t, p$ & Visit 3 & $t, p$ & Visit 4 & t, p \\
\hline \multicolumn{8}{|c|}{ Unmarried-Metformin } \\
\hline Weight & $75.22 \pm 3.56$ & $75.22 \pm 3.86$ & 0 & $75.22 \pm 3.86$ & 0 & $73.77 \pm 2.69$ & $0.97,0.36$ \\
\hline BMI & $28.55 \pm 0.88$ & $\begin{array}{l}28.55 \pm \\
0.52\end{array}$ & $0.32,0.72$ & $28.0 \pm 0.70$ & $1.47,0.09$ & $27.44 \pm 0.52$ & $3.24,0.0005^{* *}$ \\
\hline WHR & $0.84 \pm 0.03$ & $0.84 \pm 0.03$ & $0.07,0.34$ & $0.83 \pm 0.03$ & $0.07,0.34$ & $0.83 \pm 0.03$ & $0.612,0.002 * *$ \\
\hline Hirsutism & $14.66 \pm 1.14$ & $\begin{array}{l}12.77 \\
\pm 1.20 \\
\end{array}$ & $\begin{array}{l}3.05,0.001 \\
* *\end{array}$ & $10.88 \pm 0.06$ & $\begin{array}{l}7.37,0.0001 * \\
*\end{array}$ & $9.33 \pm 0.5$ & $\begin{array}{l}10.66,0.0001 * \\
*\end{array}$ \\
\hline \multicolumn{8}{|c|}{ Unmarried-Pioglitazone } \\
\hline Weight & $76.22 \pm 4.60$ & $76.33 \pm 4.12$ & $0.53,0.61$ & $77.88 \pm 4.56$ & $0.76,0.46$ & $79.33 \pm 4.41$ & $1.46,0.18$ \\
\hline BMI & $29.22 \pm 0.83$ & $29.22 \pm 0.83$ & 0 & $29.77 \pm 1.09$ & $1.21,0.01 *$ & $30.33 \pm 0.86$ & $2.77,0.001 * *$ \\
\hline WHR & $0.86 \pm 0.02$ & $0.87 \pm 0.02$ & $\begin{array}{l}0.85,0.008 * \\
*\end{array}$ & $0.88 \pm 0.02$ & $2.14,0.001 * *$ & $0.89 \pm 0.03$ & $2.30,0.001 * *$ \\
\hline Hirsutism & $14.66 \pm 1.22$ & $12.11 \pm 0.78$ & $\begin{array}{l}5.27,0.001 * \\
*\end{array}$ & $9.77 \pm 0.44$ & $5.27,0.001 * *$ & $7.55 \pm 0.52$ & $6.00,0.0001 * *$ \\
\hline \multicolumn{8}{|c|}{ Married-Metformin } \\
\hline Weight & $80.22 \pm 3.34$ & $79.88 \pm 3.01$ & $0.22,0.83$ & $78.55 \pm 3.0$ & $1.11,0.29$ & $76.88 \pm 3.58$ & $2.04,0.07$ \\
\hline BMI & $30.22 \pm 0.97$ & $30.11 \pm 0.92$ & $\begin{array}{l}2.30,0.001 * \\
*\end{array}$ & $2955 \pm 0.72$ & $1.64,0.003 * *$ & $28.88 \pm 0.78$ & $3.20,0.001 * *$ \\
\hline WHR & $0.90 \pm 0.01$ & $0.90 \pm 0.009$ & $0.488,0.34$ & $0.89 \pm 0.01$ & $0.91,0.22$ & $0.88 \pm 0.006$ & $3.71,0.001 * *$ \\
\hline Hirsutism & $16.88 \pm 0.92$ & $14.44 \pm 0.88$ & $\begin{array}{l}5.72,0.0001 \\
* *\end{array}$ & $12.22 \pm 1.09$ & $\begin{array}{l}9.76,0.0001 * \\
*\end{array}$ & $10.44 \pm 088$ & $9.76,0.0001 * *$ \\
\hline \multicolumn{8}{|c|}{ Married-Pioglitazone } \\
\hline Weight & $82.77 \pm 4.48$ & $84.0 \pm 4.33$ & $0.59,0.57$ & $84.88 \pm 5.37$ & $0.90,0.36$ & $86.44 \pm 5.01$ & $1.63,0.14$ \\
\hline BMI & $29.66 \pm 0.5$ & $30.11 \pm 0.33$ & $2.21,0.03 *$ & $30.44 \pm 0.52$ & $\begin{array}{l}3.21,0.0007 * \\
*\end{array}$ & $31.0 \pm 0.70$ & $4.61,0.0001 * *$ \\
\hline WHR & $0.91 \pm 0.01$ & $0.92 \pm 0.01$ & $\begin{array}{l}4.61,0.0001 \\
* *\end{array}$ & $0.93 \pm 0.01$ & $\begin{array}{l}3.90,0.0003 * \\
*\end{array}$ & $0.94 \pm 0.01$ & $4.71,0.0001 * *$ \\
\hline Hirsutism & $17.77 \pm 0.97$ & $13.66 \pm 1.0$ & $\begin{array}{l}8.84,0.0001 \\
* *\end{array}$ & $11.0 \pm 1.0$ & $\begin{array}{l}8.84,0.0001 * \\
*\end{array}$ & $8.33 \pm 0.5$ & $\begin{array}{l}25.92,0.0001 * \\
*\end{array}$ \\
\hline
\end{tabular}

$*=$ Significant, $* *=$ Very significant, Data are presented as Mean \pm SD, $\mathbf{n}=9$

Table 2: Comparison of clinical characteristics of PCOS.

\begin{tabular}{|lllll|}
\hline Parameters & \multicolumn{1}{c}{$\begin{array}{l}\text { Metformin } \\
\text { mean } \pm \text { SD of difference } \mathbf{V}_{\mathbf{4}}-\mathbf{V}_{\mathbf{1}}\end{array}$} & $\begin{array}{l}\text { Pioglitazone } \\
\text { mean } \pm \text { SD of difference } \mathbf{V}_{\mathbf{4}}-\mathbf{V}_{\mathbf{1}}\end{array}$ & $\mathbf{t}$ & $\mathbf{P}$ \\
\hline Unmarried (Group A) & $(-) 1.45 \pm 1.53$ & $(+) 3.11 \pm 0.78$ & 0.40 & 0.69 \\
\hline Weight & $(-) 1.11 \pm 0.60$ & $(+) 1.12 \pm 0.33$ & 000.4 & 0.99 \\
\hline BMI & $(-) 0.008 \pm 0.006$ & $(+) 0.033 \pm 0.021$ & 3.43 & $0.008^{*}$ \\
\hline WHR & $(-) 5.33 \pm 1.58$ & $(-) 7.11 \pm 1.16$ & 2.72 & $0.02^{*}$ \\
\hline Hirsutism & $(-) 3.33 \pm 1.32$ & & & \\
\hline Married (Group B) & $(+) 3.66 \pm 1.32$ & 0.53 & 0.61 \\
\hline Weight & $(-) 1.33 \pm 0.50$ & $(+) 1.33 \pm 0.5$ & - & - \\
\hline BMI & $(-) 0.014 \pm 0.008$ & $(+) 0.033 \pm 0.013$ & 3.74 & $0.005^{*}$ \\
\hline WHR & $(-) 6.44 \pm 1.58$ & $(-) 9.44 \pm 1.01$ & 4.79 & $0.001^{*}$ \\
\hline Hirsutism & & & & \\
\hline
\end{tabular}

\section{DISCUSSION}

PCOS is a common disease occurring globally in $6 \%$ to $10 \%$ of reproductive aged women. ${ }^{1,2}$ The disease has marked impact on the women health. It produces cosmetic effects which profoundly affect their psychology. ${ }^{10}$ In addition to above; PCOS is characterized by altered (anovulatory) menstrual cycle and infertility. In fact, it is one of the important causes of infertility at the young age in women. The cause of PCOS is not certainly known but endocrine disturbances particularly insulin and female sex hormone levels are 
altered. There is evidence of insulin resistance and hyperandrogenism. ${ }^{11-12}$

A number of interventions are proposed to normalize or reverse the underlined metabolic disturbances, hormonal imbalance and anovulation. These interventions include lifestyle modifications, clomiphene citrate, and gonadotripin releasing hormone agonist, glucocorticoids, antiandrogens, finastride, cimetidine, flutamide, cyperoterone acetate, insulin sensitizers and surgery. Use of metformin and pioglitazone in the treatment of PCOS is suggested because there is evidence of glucose intolerance and hyperinsulinemia and this is reversed by these drugs. ${ }^{18-21}$ Infact, in a number of reports, efficacy of both the drugs have shown in the treatment of PCOS. In 1994 metformin was tried as a first antidiabetic drug for the treatment of $\operatorname{PCOS}^{22}$ Subsequently many aspects of meformin were explored by several workers (Nestler et al, ${ }^{14}$ De Leo, ${ }^{23}$ Glueck, ${ }^{24}$ Moghetti et al, ${ }^{25}$ Shamsa Zafar ${ }^{26}$ and Pillai et $\mathrm{al}^{27}$ ) pioglitazone, another insulin sensitizer was also found to be effective in PCOS by Guido, ${ }^{28}$ Christan Haigh $^{29}$ and Romouldi. ${ }^{30}$

In the present study, subjects were divided into two groups; group A $(n=20)$, unmarried, group B $(n=20)$ married. Each group was further subdivided according to the treatment schedule. 10 unmarried women received metformin after initial evaluation and remaining 10 received pioglitazone in unmarried group. Similarly, out of 20 subjects in married group (group B), 10 received metformin and remaining 10 received pioglitazone. One patient from each subgroup dropped out from the study. Therefore 18 unmarried patients and 18 married patients completed the study.

The baseline characteristics of these subjects are shown in Table 1. As it is clear the unmarried subjects stratified into metformin and pioglitazone subdivision had comparable weight, BMI and WHRs. The FG scores were also similar in both subgroups. Thus, these clinical characteristics within the unmarried group were similar (Graph-3).

The clinical characteristics of married females varied from unmarried females. The mean weight of married subjects was significantly higher than unmarried subjects. Similarly, BMI, WHR and FG scores were also significantly higher than that of unmarried group. Therefore, for all subsequent analysis and interpretation of results these two groups were analysed separately. The within group stratification of married patients into metformin and pioglitazone dichotomy include similar baseline characteristics.

The impact of the therapy was also visible on these characteristics when metformin $500 \mathrm{mg}$ three times a day and pioglitazone $45 \mathrm{mg}$ /day were given on daily basis for 6 months. Metformin significantly reduced BMI in unmarried and married women after six months of therapy (Graph-1). This effect of metformin is explained on the basis of its anorectic effects. A number of reports showed that metformin when given to obese patients with PCOS reduced weight and BMIs due to its anorectic effect. $^{22,31}$

\section{Effect of therapy on body weight, WHR, BMI and hirsutism index}

WHR is an important indicator of obesity and risk factors for several cardiovascular disorders. ${ }^{32}$ The normal WHR for Indian patients is less than 0.85 and WHR in the present study ranged from 0.81 to 0.87 in unmarried females at baseline (no significant difference in metformin and pioglitazone treated cases.). The WHR (0.83 to 0.93 ) was significantly higher in married females as compared to unmarried females (Graph-2).

Body weight, BMI, WHR and hirsutism changes indices upon completion of therapy as shown in Table-2. Metformin significantly reduced, BMI, WHR and hirsutism index (FG score) in unmarried and married women. Compared to metformin, pioglitazone significantly increased the BMI and WHR but decreased the hirsutism index (FG score) in unmarried and married females after six month therapy. This increase in BMI and WHR can be explained on basis of fluid retaining property of pioglitazone. Pioglitazone induced weight gain has been reported in diabetics and cases with PCOS. ${ }^{33}$

The importance of hirsutism cannot be overemphasized. It has negative effect on self-esteem and self-image. ${ }^{10}$ The fear of rejection makes women reclusive and may retard their development of social skills and confidence. In a study ${ }^{32}$ women with PCOS scored lowered than women in control groups for self-esteem, body image, romantic satisfaction and involvement and comfort in social activity. It is an important factor for delayed marriage. Obviously, amelioration of these cosmetic effects is urgently needed in young women with PCOS. The fall in FG score, indicative of reduction in excessive hair growth, is an important beneficial effect of therapy. The positive effect of reduced weight and less hair growth helps achieving positive attitude towards life. ${ }^{10}$

In contrast to metformin, pioglitazone treatment resulted in slight weight gain. ${ }^{33}$ This is due to several interacting factors. In general, improvement in gycemic control with decreased glucosuria and caloric retention may result in increased weight. Several studies have shown that the weight gain with TZDs may be associated with an increase in subcutaneous adipose tissue and a concomitant decrease in visceral fat; although subcutaneous fat area decreases. This change in fat distribution may explain in part the involvement in glycemic control despite an overall increase in body weight. Fluid retention, of course, is another potential cause of increased body weight. TZDs, whether administered alone or in combination with metformin, sulfonylurea, or insulin are often accompanied by an 
increase in plasma volume. ${ }^{34}$ With an increase in plasma volume, mild to moderate oedema and $\mathrm{CHF}$ may be anticipated side effects of treatment. It is also reported that metformin may not be effective in very obese patients with $\mathrm{BMI}>37 \mathrm{Kg} / \mathrm{m}^{2} \cdot{ }^{34-37}$ None of our patients in present study have BMI $>31$. In the study of Marcondes et $\mathrm{al}^{38}$ Metformin did not change BMI. Metformin resulted in $4 \%$ reduction in waist circumference. ${ }^{39}$ Therefore from the information available in the literature shows salutary effects on weight \& BMI.

\section{CONCLUSION}

Pioglitazone significantly increased the BMI and WHR, whereas it significantly decreased FG score. On comparing metformin and pioglitazone it was observed that both the drugs equally decreased the FG score, metformin significantly decreased the BMI and WHR whereas pioglitazone significantly increased the BMI and WHR.

Funding: No funding sources

Conflict of interest: None declared

Ethical approval: The study was approved by the Institutional Ethics Committee

\section{REFERENCES}

1. Azziz R, Woods KS. The prevalence and features of PCOS in an unselected population. J Clin Endocrinol Metab. 2004;89:2745-9.

2. Hart R, Hickey M. Definitions, prevalence and symptoms of PCOS. Best Pract. Res. Clin Obstet Gynaecol. 2004;18:671-89.

3. Stein IF, Leventhal ML. Amenorrhea associated with bilateral polycystic ovaries. Am J Obstet Gynecol 1935;29:181-91.

4. Revised 2003 consensus on diagnostic criteria and long term health risks related to PCOS. Fetil Steril. 2004;81:19-25.

5. Miller K, Nixson C Boots LR. Prevalence of polycystic ovary syndrome (PCOS) in first-degree relatives of patients with PCOS. Fertil Steril 2001;75:35-8.

6. Ylid BO, Yarali HI. Glucose intolerance, insulin resistance, and hyperandrogenemia in first degree relatives of women with polycystic ovary syndrome. J Clin Endocrinol Metab. 2003;88:2031-6.

7. Escobar- Morreale HF, Luque -Ramire M, San Millan JL. The molecular-genetic basis of functional hyperandrogenism and the polycystic ovary syndrome. Endocrinol Rev. 2005;26:251-82.

8. Urbenk M, Woodroffe A, Ewens K G. Candidate gene region for polycystic ovary syndrome on chromosome. J Clin Endocrinol Metab. 2005;90:6623-29.

9. Futterweit W. Pathophysiology of polycystic ovarian syndrome. In: Redmond GP editor. Androgenic disorders. New York: Raven Press;1995:77-166.
10. Kitzinger K, Willmot J. The thief of womanhood: women's experience of polycystic ovarian syndrome. Soc Sci Med. 2002;54:349-61.

11. Burghen GA, Givens JR, Kitabchi AE. Correlation of hyperandrogenism with hypeinsulinism in polycystic ovarian disease. J Clin Endocrinol Metab 1980;50:113-6.

12. Dunaif A, Graf M, Mandeli J. Characterization of groups of hyperandrogenic women with acanthosis nigricans, impaired glucose tolerance andlor hyperinsulinimia. J Clin Metab. 1987;65:499-507.

13. Baillargeon JP, Iuorno MJ, Nestler JE. Comparison of metformin and thiazolidinediones in the management of polycystic ovary syndrome. Curr Opin Endocrinol Diabetes. 2002;9:303-11.

14. Nestler JE Jakubowicz DJ, de Vargas AF, Brik C, Quintero N, Medina F. Insulin stimulates testosterone biosynthesis by human thecal cells from women with polycystic ovary syndrome by activating its own receptor and using inositol glycan mediators as the signal transduction system. J Clin Endocrinol Metab. 1998;83:2001-05.

15. Waist circumference and waist-hip ratio report of a WHO expert consultation GENEVA, 8-11 December 2008.

16. Park K. Epidemiology of chronic non communicable diseases and conditions. Park's textbook of preventive and social medicine. $20^{\text {th }}$ edition. Jabalpur : M/s Banarsidas Bhanot; 2009:348.

17. Ferriman D, Gallwey JD. Clinical assessment of body hair in women. J Clin Endocrinol Metab 1961;21:1440-8.

18. Glueck CJ, Moreira A, Goldenberg N, Wang P. Pioglitazone and metformin in obese women with PCOS not optimally responsive to metformin. Hum Reprod. 2003;18(8):1618-25.

19. Gonzalez, Ortege, Luna S, Hernander L, Crespo G, Aguayo P. Responses of serum androgen and insulin resistance to metformin and pioglitazone in obese, resistant women with PCOS. J Clin Endocrinol Metab. 2005;90(3):1360-5.

20. Steiner CA, Janez A, Jensterle M, Reisinger K, Pfutzner. Impact of treatment with rosiglitazone or metformin on biomarkers for insulin resistance and metabolic syndrome in patients with PCOS. J Diabe Scinece Techno. 2007;1(2):211-5.

21. Cetinkalp, Svki, Karadeniz, Muammer, Erdogan. The effects of rosiglitazone, metformin and estradiol_cyproterone acetate on lean patients with PCOS. Endocrinologist. 2009;19(3):94-7.

22. Velazquez EM, Mendoza S, Hamer T. Metformin therapy in PCOS reduces hyperinsulinemia, insulin resistance, hyperandrogenemia and systolic blood preasure, while facilitating normal menses and pregnancy. Metbolism. 1994;43:647-54.

23. De Leo V, la Marca E, Morgante G, Cianci A. Effects of Metformin on gonadotropin induced ovulation in women with PCOS. Fertil Steril 1999;72:282-5. 
24. Glueck CJ. Metformin induced resumption of normal menses in 39 of $43(91 \%)$ previously amenorrheic women with polycystic ovary syndrome. Metabolism. 1999;48:511-9.

25. Moghetti P, Castello R, Negri C. Metformin effects on clinical features endocrine and metabolic profiles and insulin sensitivity in PCOS: A randomized double blind placebo controlled 6 month trial, followed by open long term clinical evaluation. J Clin Endocrinol Metab. 2000;85:139-46.

26. Zafar S. Role of metformin in correcting hyperinsulinemia, menstrual irregularity and anovulation in PCOS. J Ayub Med. Coll Abbottabad. 2006;17(4):120-5.

27. Pillai A, Bang H, Green C. Metformin and glitazones: Do they really help PCOS patients ? J Fam Proct. 2007;56(6):444-53.

28. Gudio M, Romouldi D, Suriano R, Giuliani M, Apa $\mathrm{R}$. Effect of pioglitazone treatment on the adrenal androgen response to corticotrophin in obese patients with PCOS. Hum Reprod. 2004;19(3):5349.

29. Haigh. Pioglitazone treatment demonstrated improvements in insulin sensitivity. J Clin Endocrinol Metab. 2009;94:469-76.

30. Romualdim D, Guide M, Ciamplesi M, Gintiani M, Leoni F, Perri C. Selective effects of pioglitazone on insulin and androgen abnormalities in normal-hyperinsulinemic obese patients with PCOS. Hum Reprod. 2003;18(6):1210-8.

31. Nestler JE Jakubowicz DJ. Lean women with PCOS respond to insulin reduction with decrease in ovarian P450 C17 alpha activity and serum androgens. J Clin Endocrinol Metab. 1997;82:4075-9.

32. Magnotti M, Futterweit W. Obesity and the PCOS. Med Clin N Am. 2007;91:1151-68.
33. Gonzalez O, Luna S, Hernander L, Crespo G, Aguayo P. Responses of serum androgen and insulin resistance to metformin and pioglitazone in obese, resistant women with PCOS. J Clin Endocrinol Metab. 2005;90(3):1360-5.

34. Avandia (rosiglitazone maleate). Philadelphia, PA: Glaxo Smith Kline Pharmaceuticals; 2000.

35. Gambineri A Pelusi C, Vicennti V, Pasquali R. Obesity and the PCOS. Int J Obes Relat Metab Disord. 2002;883-96.

36. Ehrmannn DA. Effects of metformin on insulin secretion, insulin action and ovarian steroidogenesis in women with polycystic ovarian syndrome. J Clin Endocrinol Metab. 1997;82:524-30.

37. Fleming R, Hopkinson ZE, Wallace AM, Greer IA, Satttar N. Ovarian function and metabolic factors in women with oligomenorrhoea treated with metformin in a randomized double blind, placebo controlled trial. J Clin Endocrinol Metab 2002;87:569-74.

38. Marcondes JA, Yamashita SA, Maciel GA, Boacat EC, Halpem A. Metformin in normal weight hirsute women with PCOS with normal insulin sensitivity. Gynecol Endocrinol. 2007;23(5):273-8.

39. Hoeger K, Davidson K, Kochman L, Cherry T, Kopin L, Guzick DS. Impact of metformin, oral contraceptives and lifestyle modification on PCOS in obese adolescent women in two randomized placebo controlled clinical trials. J Clin Endocrinol Metab. 2008;93(11):4299-30.

Cite this article as: Tanwar S, Khilnani G. A clinical comparative study on the effects of metformin and pioglitazone on clinical symptoms in cases of polycystic ovarian syndrome (PCOS). Int J Basic Clin Pharmacol 2016;5:98-104. 